\title{
Silicon-on-Insulator CWDM Power Monitor/Receiver With Integrated Thin-Film InGaAs Photodetectors
}

\author{
J. Brouckaert, Student Member, IEEE, G. Roelkens, Member, IEEE, S. K. Selvaraja, Student Member, IEEE, \\ W. Bogaerts, Member, IEEE, P. Dumon, Member, IEEE, S. Verstuyft, D. Van Thourhout, Member, IEEE, and
} R. Baets, Fellow, IEEE

\begin{abstract}
In this letter, we demonstrate a compact and cost-effective four-channel demultiplexer with integrated photodetectors for application in coarse wavelength-division-multiplexing systems. The device consists of a silicon-on-insulator planar concave grating (PCG) demultiplexer and heterogeneously integrated InAlAs-InGaAs metal-semiconductor-metal photodetectors, and has a footprint of only $0.1 \mathrm{~mm}^{2}$. The PCG and integrated photodetectors have a responsivity of $0.6 \mathrm{~A} / \mathrm{W}$ for TE-polarized light. The integrated device has an optical crosstalk of $-25 \mathrm{~dB}$.
\end{abstract}

Index Terms-Demultiplexing, heterogeneous integration, photodetector, silicon-on-insulator (SOI).

\section{INTRODUCTION}

$\mathbf{C}$ OARSE wavelength-division-multiplexing (CWDM) systems have a channel spacing of typically $20 \mathrm{~nm}$ on the International Telecommunication Union (ITU) wavelength grid, and are designed for use with uncooled transmitter lasers. CWDM makes it possible to increase the capacity of a fiber optic link without installing expensive dense WDM (DWDM) systems. Currently, the majority of CWDM transceivers are based on thin-film filter (TFF) technology. The main drawback to TFFs lies in the assembly costs of the discrete components and the difficulty of integration with lasers and detectors. Highly integrated modules are a key part of the solution to mass produce these CWDM transceivers in a cost-effective way when reducing size and increasing reliability. Both silica-based CWDM receivers and large-core silicon-on-insulator (SOI) transceivers with hybridized detectors and lasers have been proposed [1], [2]. However, the integration density of these transceivers remains limited due to, first of all, the large waveguide bend radius in these material systems, and second, the

Manuscript received March 11, 2009; revised June 15, 2009. First published July 24, 2009; current version published September 18, 2009. This work was supported by the Institute for the Promotion of Innovation through Science and Technology (IWT Vlaanderen) under the SBO epSOC project. The work of G. Roelkens was supported by the Fund for Scientific Research.

J. Brouckaert, G. Roelkens, S. K. Selvaraja, D. Van Thourhout, and R. Baets are with the Department of Information Technology, Ghent University, Interuniversity Microelectronics Center, B-9000 Gent, Belgium (e-mail: joost.brouckaert@intec.ugent.be; gunther.roelkens@intec. ugent.be; shankar@intec.ugent.be; dries.vanthourhout@intec.ugent.be; roel.baets@intec.ugent.be).

W. Bogaerts, P. Dumon, and S. Verstuyft are with the Photonics Research Group, Department of Information Technology, Ghent University, Interuniversity Microelectronics Center, B-9000 Gent, Belgium (e-mail: wim.bogaerts@intec.ugent.be; pieter.dumon@intec.ugent.be; steven. verstuyft@intec.ugent.be).

Color versions of one or more of the figures in this letter are available online at http://ieeexplore.ieee.org.

Digital Object Identifier 10.1109/LPT.2009.2027999

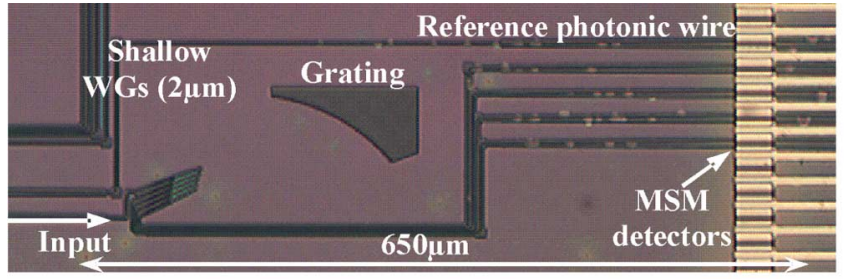

(a)

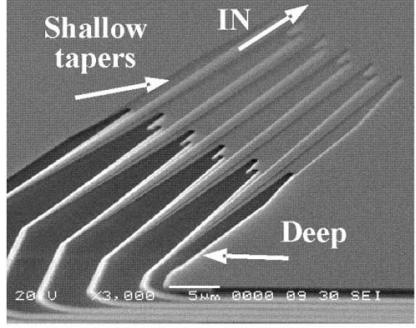

(b)

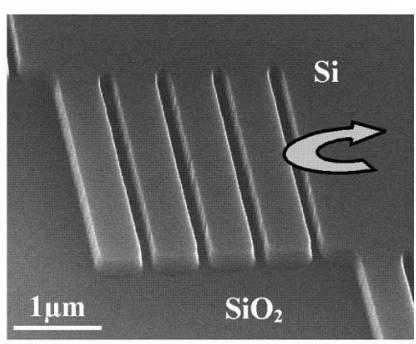

(c)
Fig. 1. (a) Top view picture of the CWDM receiver. (b) SEM picture of the double adiabatic tapers. (c) SEM picture of a DBR grating reflector.

large waveguide pitch needed for the hybridization of preprocessed lasers and detectors.

In this letter, we demonstrate a compact CWDM demultiplexer consisting of a four-channel planar concave grating (PCG) fabricated on a nanophotonic SOI platform and heterogeneously integrated thin-film III-V photodetectors. This device can be used either as an integrated CWDM power monitor or receiver. SOI photonic wire waveguides with a typical bend radius of a few micrometer allow to fabricate very compact demultiplexers, making use of CMOS compatible processes [3]. The detector integration approach is based on the bonding of unprocessed III-V dies onto the SOI waveguide wafer [4]. After bonding, the detectors can be fabricated on a wafer scale and lithographically aligned onto the output waveguides of the demultiplexer. This integration schema avoids time-consuming and alignment-critical hybridization of preprocessed detectors [1], [2] while making very small detector pitches of $25 \mu \mathrm{m}$ possible.

\section{DESIGN AND FABRICATION}

The fabricated CWDM power monitor/receiver is shown in Fig. 1(a). It is processed on a 200-mm SOI wafer with a 220-nm-thick Si waveguide layer. A PCG demultiplexes the input signal into four output channels with a spacing of $20 \mathrm{~nm}$ around a designed central wavelength of $1550 \mathrm{~nm}$. Thin-film 


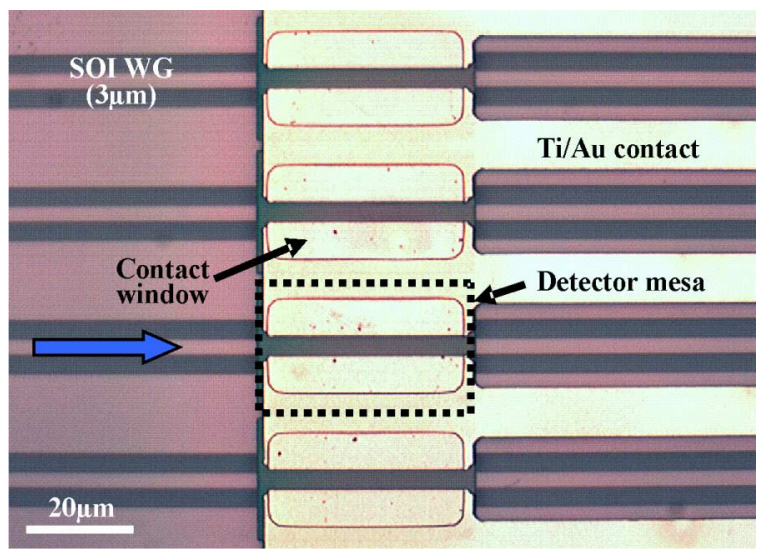

Fig. 2. Top view of three MSM detectors on top of 3- $\mu \mathrm{m}$-wide SOI waveguides.

InAlAs-InGaAs metal-semiconductor-metal (MSM) photodetectors with a length of $40 \mu \mathrm{m}$ are heterogeneously integrated onto the output waveguides. The total footprint of the demultiplexer, including photodetectors, is only $\sim 650 \times 150 \mu \mathrm{m}^{2}$.

The design of the PCG is based on the Rowland geometry and clearly described elsewhere [3], [5]. The entrance and exit waveguides are positioned on a circle with a radius of $94 \mu \mathrm{m}$, and the curved grating sits on a $188-\mu$ m-radius circle. The order of diffraction is 10 . A double adiabatic taper is used for the transition from the 500-nm-wide deeply etched photonic wire access waveguides to the $2-\mu \mathrm{m}$-wide shallowly etched (70 $\mathrm{nm}$ deep) entrance and exit waveguides on the Rowland circle [Fig. 1(b)]. The spacing between the centers of the exit waveguides along the Rowland circle is $5 \mu \mathrm{m}$. The incident angle is $41^{\circ}$ and the diffraction angle is $37^{\circ}$. This configuration results in a linear dispersion of $0.25 \mu \mathrm{m} / \mathrm{nm}$ [3]. Structures were defined with a 193-nm deep-UV (DUV) lithography, and transferred into the silicon using inductively coupled plasma (ICP) reactive ion etching (RIE) [6]. To increase the reflectivity of the grating, we replaced the 31 flat facets with second-order distributed Bragg (DBR) reflectors [Fig. 1(c)]. The DBRs have four 600-nm-wide periods consisting of a 130-nm-wide deeply etched trench and a 470-nm-wide unetched region. The theoretical reflection loss is lower than $0.5 \mathrm{~dB}$ in the $1.5-1.6 \mu \mathrm{m}$ wavelength range. The demultiplexer was first characterized before detector integration [5]. The on-chip loss is $1.9 \mathrm{~dB}$ for the central channels, with a $1.7 \mathrm{~dB}$ nonuniformity across the four 20-nm-spaced wavelength channels. The crosstalk is better than $-25 \mathrm{~dB}[5]$.

We integrated MSM photodetectors [4], [7] on the $25-\mu \mathrm{m}$-spaced output waveguides. Light from a $3-\mu \mathrm{m}$-wide, 220-nm deeply etched SOI waveguide is evanescently coupled into the thin-film detector waveguide on top of it (Fig. 2). The SOI waveguide and the detector are separated by a 100-nm-thick divinyl-tetramethyldisiloxane-benzocyclobutene (DVS-BCB) bonding layer. The detector layer stack has a height of $205 \mathrm{~nm}$, and consists of a 40-nm-thick InAlAs Schottky barrier enhancement layer (SBEL) on top of a 20 -nm-thick InAlGaAs graded layer on a $145-\mathrm{nm}$ InGaAs absorption layer. Two coplanar Ti-Au coplanar Schottky contacts with a $3 \mu \mathrm{m}$ spacing are processed onto the SBEL [4]. The fabrication starts with bonding unprocessed III-V

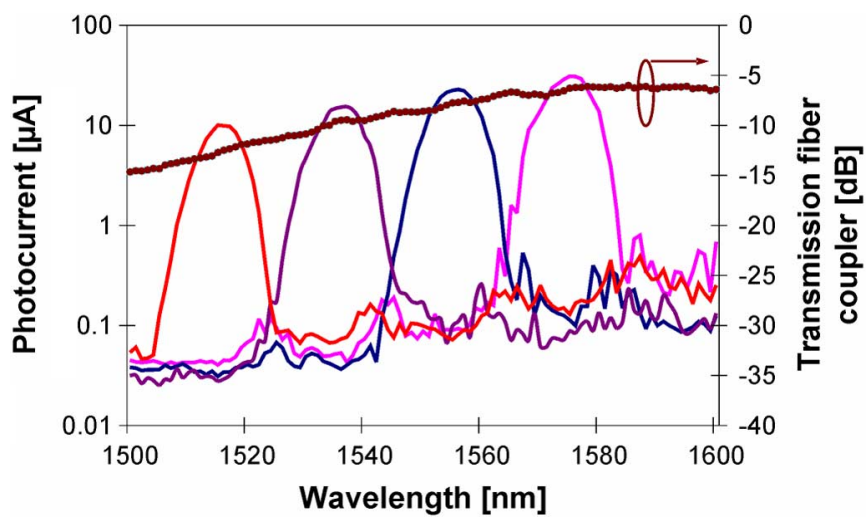

Fig. 3. Photocurrent spectrum of the CWDM receiver for TE-polarized light on the left axis and transmission of a fiber coupler on the right axis.

dies (epitaxial layers down) onto the SOI waveguide substrate using DVS-BCB [7]. After bonding, the sample is cured in a nitrogen environment at a temperature of $250{ }^{\circ} \mathrm{C}$ for $1 \mathrm{~h}$. As the dies are unprocessed, a strict alignment accuracy is not necessary. After the removal of the InP substrates and etch-stop layers, we obtain defect-free unprocessed epilayers bonded onto the SOI substrate. Processing was done on $2 \mathrm{~cm} \times 2 \mathrm{~cm}$ SOI samples, but it could be carried out on an SOI wafer scale. Then the individual detector mesas (Fig. 2) are etched down to the DVS-BCB bonding layer using nonselective wet etching. A DVS-BCB insulation layer is spun on top and contact windows are opened. Finally, the coplanar Schottky contacts are defined. The detectors have a length of $40 \mu \mathrm{m}$, a pitch of $25 \mu \mathrm{m}$, a responsivity of $1.0 \mathrm{~A} / \mathrm{W}$ at $\lambda=1.55 \mu \mathrm{m}$, and a dark current of $\sim 5 \mathrm{nA}$ at $5 \mathrm{~V}$ bias [7]. The dark current increases only by a factor $\sim 15$ (still below $100 \mathrm{nA}$ ) when the temperature increases from $20^{\circ} \mathrm{C}$ to $75^{\circ} \mathrm{C}$, making uncooled operation possible.

\section{MEASUREMENT Results}

To characterize the demultiplexer with integrated detectors, we coupled TE-polarized light from a single-mode fiber into the nanophotonic input waveguide using a shallowly etched 1-D grating (fiber coupler) [8]. The measured spectral response of the receiver (TE polarization) is shown in Fig. 3. The transmission spectrum of the fiber coupler is shown in the right axis. The minimum fiber to waveguide coupling loss was estimated to be $6 \mathrm{~dB}$ at a wavelength of $1580 \mathrm{~nm}$. Due to the large birefringence of the nanophotonic waveguides, all components including PCG, fiber coupler, and photodetectors are designed for TE polarization only. To obtain polarization-independent operation, a polarization diversity configuration using 2-D fiber couplers could be implemented [9]. The optical crosstalk is $-25 \mathrm{~dB}$ and no significant deterioration of PCG characteristics were observed after detector integration. The presence of a DVS-BCB layer $(n=1.54)$ slightly decreases the refractive index contrast of the DBR facets, resulting in a minor increase of grating reflection loss by $0.2 \mathrm{~dB}$ on average in the $1.5-1.6 \mu \mathrm{m}$ wavelength range. The channel nonuniformity is mainly caused by the transmission spectrum of the fiber coupler, as can be seen in Fig. 3. The detector efficiency is almost constant in the considered wavelength range [4], [5]. 


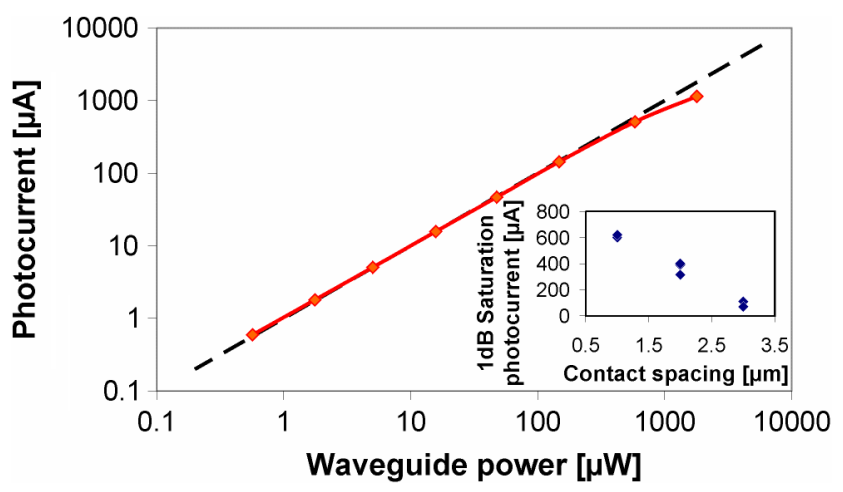

Fig. 4. Measured photocurrent as a function of optical power of a 40- $\mu \mathrm{m}$-long detector. Schottky contact spacing is $1 \mu \mathrm{m}$. The dashed line represents a responsivity of $1 \mathrm{~A} / \mathrm{W}$. (Inset) Measured $1 \mathrm{~dB}$ saturation photocurrent as a function of contact spacing for seven different samples/processing runs.

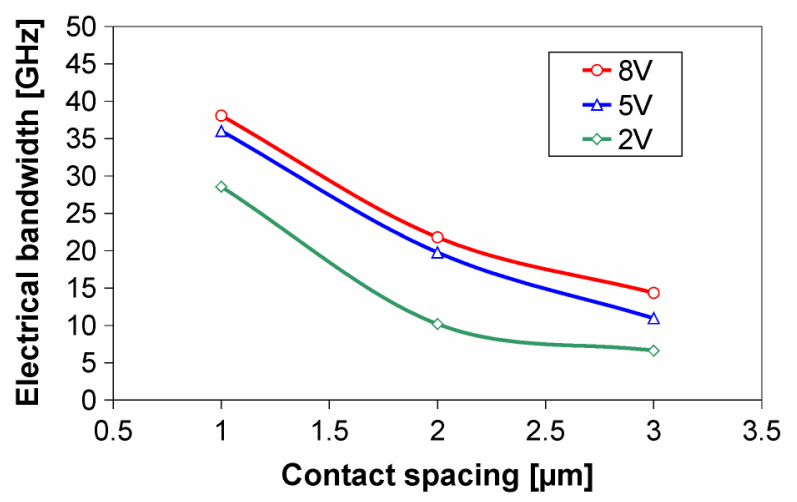

Fig. 5. Simulated electrical bandwidth as a function of contact spacing for different bias voltages. The waveguide width and contact spacing are equal.

The power budget can be calculated as follows. By finetuning the fiber coupler period, it is possible to make the central PCG wavelength and the maximum fiber coupler transmission coincide at $\lambda=1.55 \mu \mathrm{m}$. In this case, the total loss for the central channels is about $6 \mathrm{~dB}$ (fiber coupler loss) $+2 \mathrm{~dB}$ (on-chip PCG loss). Taking into account a detector responsivity of $1 \mathrm{~A} / \mathrm{W}$ at $1.55 \mu \mathrm{m}$, the total responsivity for the central channels is $\sim 0.15 \mathrm{~A} / \mathrm{W}$. However, as the main loss contribution is due to fiber coupling loss, the total responsivity of the receiver could be considerably increased (up to $0.6 \mathrm{~A} / \mathrm{W}$ ) by using a more efficient fiber to waveguide coupling approach [10], [11].

For use as an optical channel performance monitor (power monitor), a tapped signal from the main CWDM data link can be coupled into the chip using a single-mode fiber. For these applications, a linear behavior is important. Fig. 4 shows the measured photocurrent of a detector with a Schottky contact spacing and SOI waveguide width of $1 \mu \mathrm{m}$ at $5 \mathrm{~V}$ bias as a function of waveguide power. As can be seen, an effective linear behavior is obtained over a large power range. The $1 \mathrm{~dB}$ saturation photocurrent is $610 \mu \mathrm{A}$ [averaged measurement over two different samples, Fig. 4 (inset)]. Fig. 4 (inset) shows this $1 \mathrm{~dB}$ saturation current as a function of Schottky contact spacing. For wider contact spacing, the output saturates faster due to carrier screening effects. For use as a CWDM receiver, a large bandwidth is important. Simulations of the electrical bandwidth as a function of contact spacing are shown in Fig. 5. These are 2-D finite-element simulations, which take into account a $50 \Omega$ termination. Due to the small capacitance of the compact device, the bandwidth is transit-time limited for contact spacings above $1 \mu \mathrm{m}$. High-speed operation $(>30 \mathrm{GHz}$ ) is possible by decreasing the contact spacing and waveguide width down to $1 \mu \mathrm{m}$. Measurements need to be carried out to confirm these results.

\section{CONCLUSION}

We demonstrated a four-channel CWDM power monitor/receiver that consists of an echelle grating demultiplexer fabricated on an SOI substrate and heterogeneously integrated InGaAs photodetectors. These devices could be mass produced in a cost-effective way due to the wafer scale processing of passive waveguide circuitry and III-V photodetectors on low-cost, highquality SOI substrates using CMOS fabrication tools. The use of photonic wire waveguides and heterogeneously integrated photodetectors on a $25 \mu \mathrm{m}$ pitch resulted in a very compact device with of footprint of $0.1 \mathrm{~mm}^{2}$.

\section{REFERENCES}

[1] Y. Doi, M. Ishii, S. Kamei, I. Ogawa, S. Mino, T. Shibata, Y. Hida, T. Kitagawa, and K. Kato, "Flat and high responsivity CWDM photoreceiver using silica-based AWG with multimode output waveguides," Electron. Lett., vol. 39, no. 22, pp. 1603-1604, Oct. 2003.

[2] B. J. Luff, D. Feng, D. C. Lee, W. Qian, H. Liang, and M. Asghari, "Hybrid silicon photonics for low-cost high-bandwidth link applications," Adv. Opt. Technol., vol. 2008, pp. 245131-1-245131-6, 2008, (doi:10.1155/2008/245131B).

[3] J. Brouckaert, W. Bogaerts, P. Dumon, D. Van Thourhout, and R. Baets, "Planar concave grating demultiplexer fabricated on a nanophotonic silicon-on-insulator platform," J. Lightw. Technol., vol. 25, no. 5, pp. 1269-1275, May 2007.

[4] J. Brouckaert, G. Roelkens, D. Van Thourhout, and R. Baets, "Thin-film III-V photodetectors integrated on silicon-on-insulator photonic ICs," J. Lightw. Technol., vol. 25, no. 4, pp. 1053-1060, Apr. 2007.

[5] J. Brouckaert, W. Bogaerts, S. Selvaraja, P. Dumon, R. Baets, and D. Van Thourhout, "Planar concave grating demultiplexer with high reflective Bragg reflector facets," IEEE Photon. Technol. Lett., vol. 20, no. 4, pp. 309-311, Feb. 15, 2008.

[6] S. K. Selvaraja, P. Jaenen, S. Beckx, W. Bogaerts, P. Dumon, D. Van Thourhout, and R. Baets, "Silicon nanophotonic wire structures fabricated by $193 \mathrm{~nm}$ optical lithography," in Proc. 20th Annu. Meeting IEEE LEOS 2007, pp. 48-49.

[7] J. Brouckaert, G. Roelkens, D. Van Thourhout, and R. Baets, "Compact InAlAs-InGaAs metal-semiconductor-metal photodetectors integrated on silicon-on-insulator waveguides," IEEE Photon. Technol. Lett., vol. 19, no. 19, pp. 1484-1486, Oct. 1, 2007.

[8] W. Bogaerts, D. Taillaert, B. Luyssaert, P. Dumon, J. Van Campenhout, P. Bienstman, D. Van Thourhout, R. Baets, V. Wiaux, and S. Beckx, "Basic structures for photonic integrated circuits in silicon-on-insulator," Opt. Express, vol. 12, pp. 1583-1591, 2004.

[9] W. Bogaerts, D. Taillaert, P. Dumon, D. Van Thourhout, and R. Baets, "A polarization-diversity wavelength duplexer circuit in silicon-on-insulator photonic wires," Opt. Express, vol. 15, pp. 1567-1578, 2007.

[10] G. Roelkens, D. Van Thourhout, and R. Baets, "High efficiency grating couplers between silicon-on-insulator waveguides and perfectly vertical optical fibers," Opt. Lett., vol. 32, pp. 1495-1497, 2007.

[11] S. J. McNab, N. Moll, and Y. A. Vlasov, "Ultra-low loss photonic integrated circuit with membrane-type photonic crystal waveguides," Opt. Express, vol. 11, pp. 2927-2939, 2003. 